\title{
Nanocube Imprint Lithography
}

\author{
Harshal Agrawal and Erik C. Garnett* \\ Cite This: https://dx.doi.org/10.1021/acsnano.0c04793 \\ Read Online
}

ACCESS | Lلll Metrics \& More | 回 Article Recommendations

ABSTRACT: In recent years, imprint lithography has emerged as a promising patterning technique capable of high-speed and volume production. In this work, we report highly reproducible one-step printing of metal nanocubes. A dried film of monocrystalline silver cubes serves as the resist, and a soft polydimethylsiloxane stamp directly imprints the final pattern. The use of atomically smooth and sharp faceted nanocubes facilitates the printing of high-resolution and well-defined patterns with face-to-face alignment between adjacent cubes. It also permits digital control over the line width of patterns such as straight lines, curves, and complex junctions over an area of several square millimeters. Single-particle lattices as well as three-dimensional nanopatterns are also demonstrated with an aspect ratio up to 5 in the vertical direction. The high-fidelity nanocube patterning combined with the previously demonstrated epitaxial overgrowth can enable curved (single) crystals from solution at room temperature or highly efficient transparent conductors.

KEYWORDS: colloidal ink, nanocubes, assembly, large-scale printing, patterning, PDMS

M icro- and nanolithography have been employed for decades to realize patterned electronic and photonic devices. $^{1-3}$ Electron beam lithography (EBL) provides excellent fidelity and precise positioning on the substrate; however, it is expensive, with multiple steps, and scalability is a major deterring factor. Successive etching steps used for pattern transfer can also roughen the surface and degrade the material quality by introducing defects. Other patterning techniques have been developed to avoid the etching step including microcontact printing, ${ }^{4-6}$ dip-pen lithography, ${ }^{7}$ inkjet printing, ${ }^{8}$ scanning probe lithography, ${ }^{9,10}$ and template-directed assembly, ${ }^{11-13}$ but these all suffer from problems with either resolution or scalability. Imprint lithography has the potential to simultaneously deliver surface patterning with sub $10 \mathrm{~nm}$ feature size while delivering highthroughput over large areas. ${ }^{14-22}$ Additionally, it is relatively cheap, material-efficient, and free of vacuum-based high-end equipment and holds the promise of bringing patterned $3 \mathrm{D}$ nanoarchitectures to life. ${ }^{23-26}$

Soft imprint lithography utilizes an elastomeric stamp having trenches or ridges in the desired dimensions and shape for pattern transfer to the underlying film. ${ }^{24,27-29}$ This simple yet powerful embossing concept has enabled efficient plasmonic back-reflector solar cells, ${ }^{30}$ periodic arrays as antireflection coating, ${ }^{31}$ template-confined growth of crystals, ${ }^{32,33}$ surfaceenhanced Raman spectroscopy substrates, ${ }^{34}$ transparent conductors, $^{35-38}$ nanobatteries, ${ }^{26}$ and other nanoelectronic devices such as organic field-effect transistors. ${ }^{39}$ Polycrystalline spherical nanoparticles of $\mathrm{Ag}, \mathrm{Au}, \mathrm{TiO}_{2}$, and indium tin oxide (ITO) have been used as the ink for patterning and are subsequently sintered at high temperatures to form a crystal. ${ }^{26}$
The thermal fusing step combined with the gaps inherent in close-packed spheres can lead to porosity, shrinkage, and even cracking. The sintering temperatures are also often high (450 $\left.{ }^{\circ} \mathrm{C}\right),{ }^{26,30,40,41}$ which is still an impeding factor for true integration of imprint lithography of nanoparticle-based devices on heat-sensitive flexible polymeric substrates essential for high-throughput roll-to-roll processing.

In this article, we report the printing of complex nanopatterns using monocrystalline $\mathrm{Ag}$ nanocubes capped with polyvinylpyrrolidone (PVP) directly as an imprint resist. The use of atomically smooth and single-crystalline nanocubes as ink facilitates alignment between adjacent particles and enables high-precision patterns with a smooth surface as well as sharp, well-defined edges and essentially no void space. Polydimethylsiloxane (PDMS) is used as the stamp to pattern and print the cubes over an area of up to a few $\mathrm{mm}^{2}$, which is only limited by the setup used. The technique proposed is highly reproducible and enables directed assembly of colloidal particles in profiles such as curves and lines on rigid as well as flexible substrates. We show that it is possible to control the line width of patterns with digital accuracy $(1,2,3, \ldots, n$ particles wide) and print patterns with an aspect ratio up to 5 in the vertical direction. The patterning is largely independent of the material properties of the nanocubes and thus broadly

Received: June 8, 2020

Accepted: August 3, 2020

Published: August 3, 2020 


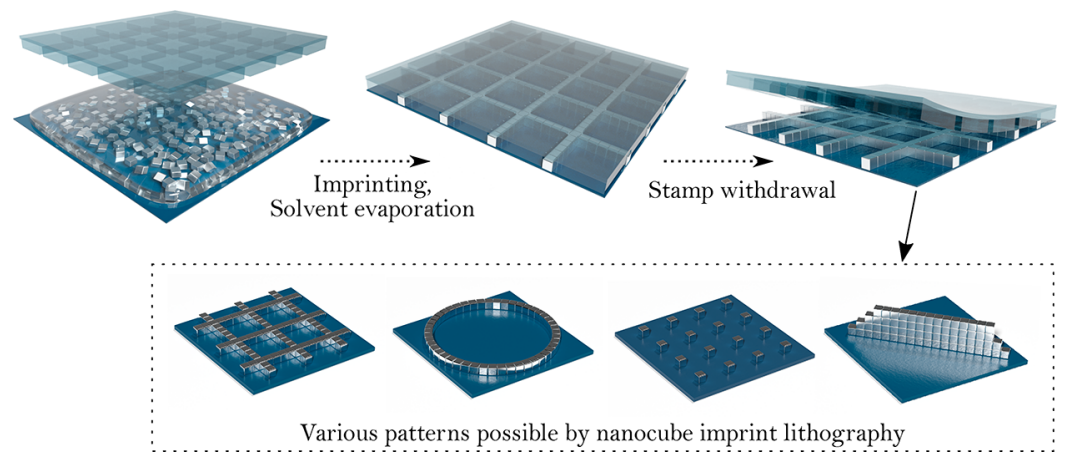

Figure 1. Schematic of the nanocube imprint lithography process.

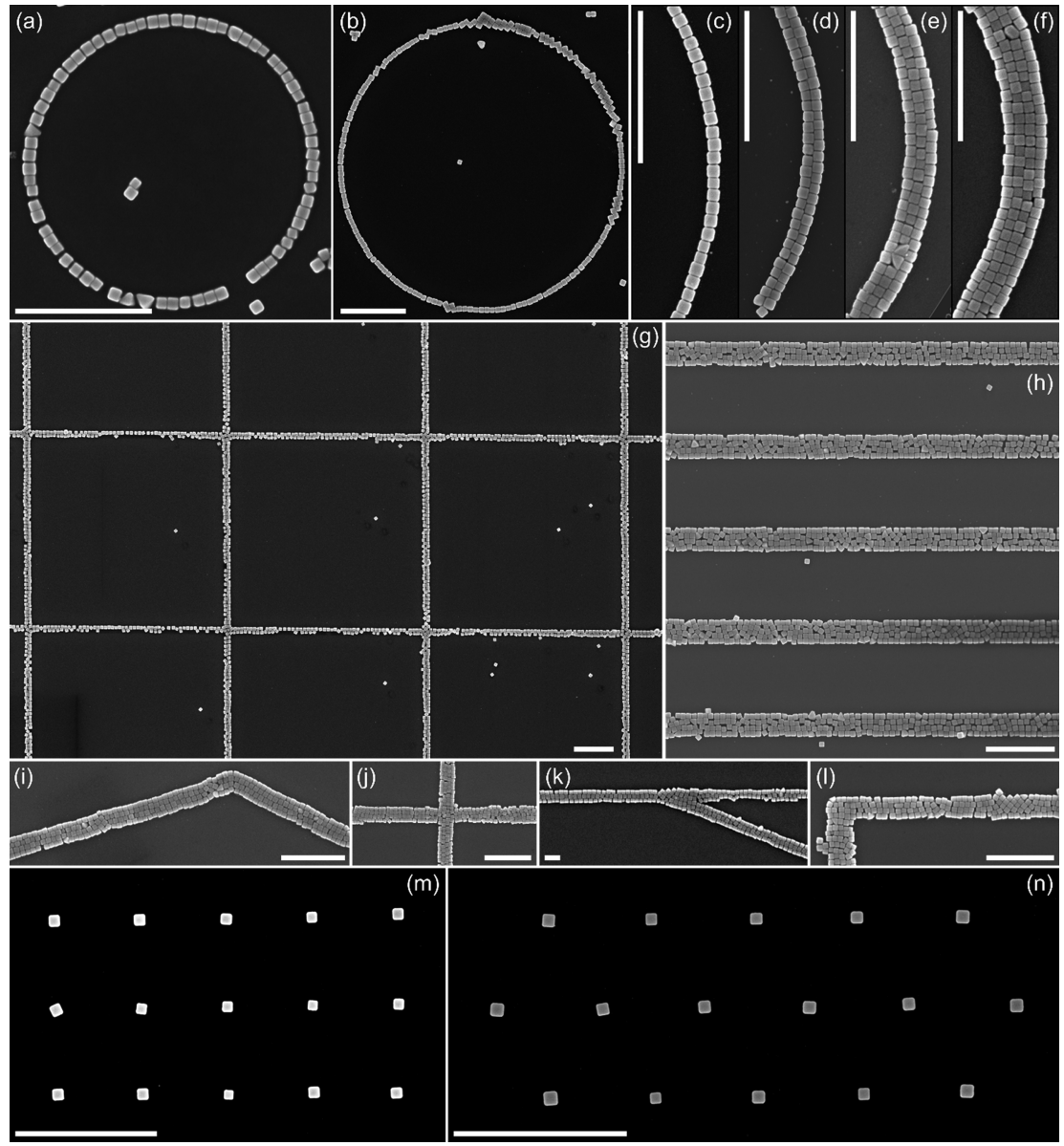

Figure 2. A variety of printed patterns of Ag nanocubes demonstrating the potential of nanocube imprint lithography. (a and b) Circles of 1 and $2 \mu \mathrm{m}$ radii of curvature, respectively. (c-f) Arcs of $2 \mu \mathrm{m}$ radius of curvature with a line width of $1,2,3$, and 4 cubes, respectively. (g) Cubes printed in a grid pattern with a pitch of $5 \mu \mathrm{m}$. (h) Gratings of cubes with a pitch of $1.4 \mu \mathrm{m}$. (i) An acute angle turn. (j) A four-way junction. (k) A three-way junction. (1) Sharp $90^{\circ}$ turn (corner). (m) Cubic and (n) hexagonal superlattices of Ag cubes. All scale bars are 1 $\mu \mathrm{m}$ in length.

applicable to a variety of materials. Finally, the crystallographic alignment inherent to nanocube assembly combined with the patterning demonstrated here is promising for printing strainand dislocation-free single crystals with arbitrary nanopatterns at room temperature. ${ }^{42,43}$

\section{RESULTS AND DISCUSSION}

The process flow of nanocube imprint lithography is illustrated in Figure 1. The process requires three key components. First is the substrate on which the pattern needs to be printed. It can be any rigid material such as silicon $(\mathrm{Si})$, glass, quartz, or any flexible material such as cellulose acetate (projector transparency), polyimide film (Kapton tape), or a polyethylene terephthalate (PET) foil. Second is the nanocube ink of the desired material dispersed in a solvent. Colloidal nanocube synthesis has been reported in the literature for a variety of materials, for example, in the class of metals $\mathrm{Ag}, \mathrm{Au}, \mathrm{Cu}, \mathrm{Ni}$, $\mathrm{Pd}, \mathrm{Pt}$, and $\mathrm{Rh}^{44-48}$ semiconductors $\mathrm{PbS}, \mathrm{Cu}_{2} \mathrm{O}, \mathrm{FeS}_{2}, \mathrm{Fe}_{2} \mathrm{O}_{3}$, $\mathrm{CsPbBr}, \mathrm{CsPbI}_{3}$, and $\mathrm{CsPCl}_{3} ;{ }^{49-53}$ and dielectrics $\mathrm{BiFeO}_{3}$, $\mathrm{SrTiO}_{3}$, and $\mathrm{CeO}_{2} .{ }^{54-57}$ We have used $\mathrm{Ag}$ nanocubes with an edge length of $75 \mathrm{~nm}$ and dispersed in ethanol as the colloidal 


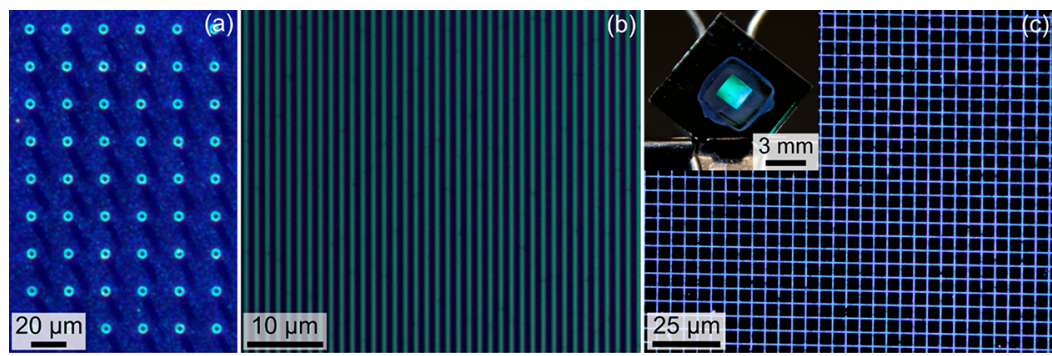

Figure 3. Potential for large-scale imprinting. (a) Optical micrograph of arrays of circles of Ag cubes. (b) Optical micrograph of gratings of commercially available $50 \mathrm{~nm}$ silica spheres. (c) Optical image of the printed grid of Ag nanocubes. The inset depicts the Si substrate from which the optical image was taken, and the center of the substrate is reflecting the bright colors because of the diffraction of light due to the printed grid.

ink, as shown in Figure S1. The third component is an elastomeric stamp (also called a template) that has the desired patterns in the form of nanotrenches. These trenches are made by casting the polymer on the Si master (Figures S2 and S3) that has the inverse pattern made by EBL and plasma etching (refer to Supporting Information for the fabrication details of the master and the stamp). The PDMS stamps (Figure S4) have trenches forming different patterns: circles with various radii of curvature, gratings, and grids, all with various line width, pitch, and depth (see Table S1 for all parameters).

In a typical imprinting process, the surface of the substrate ( $\mathrm{Si}$, Figure S5) is first activated with UV-ozone treatment to ensure even wetting over the entire substrate surface. Then the nanocube ink (Ag nanocubes dispersed in ethanol) is dispensed onto the substrate at room temperature. The solvent is allowed to dry completely, leaving a film of nanocubes, which is referred to as a nanocube resist (Figure S6). Subsequently, pure solvent (ethanol) is dispensed onto the same spot over the dried film of cubes, and before the solvent evaporates, the elastomeric stamp is pressed onto the substrate. The temperature of the substrate is raised to $40{ }^{\circ} \mathrm{C}$ and held there for $20 \mathrm{~min}$ to ensure complete solvent evaporation. Lastly, the stamp is withdrawn with a motorized control $(200 \mu \mathrm{m} / \mathrm{min})$. This leaves behind nanocube patterns on the substrate. The setup used for the nanocube imprint lithography is shown in Figure S7, and a video of the process is available in the Supporting Information.

The complete drying to form a nanocube resist layer followed by rewetting with pure solvent was found to be essential for reproducible, high-fidelity patterning. Direct printing of the colloidal solution before drying led to highly nonuniform patterns, with many trenches missing particles even when very high colloidal concentrations were used (Figure S8). On the other hand, imprinting on the dry nanocube resist (without rewetting) led to many particles remaining on the substrate in areas outside the targeted pattern (Figure S9). It therefore appears that the drying step binds the particles to the substrate in order to drastically increase the effective concentration, while the addition of solvent afterward allows for lateral mobility on the substrate surface without complete redispersion. PDMS is known to be permeable to a variety of solvents including ethanol, and hence the solvent can evaporate through PDMS during the heating step. ${ }^{58}$ Excess cubes stuck to the surface of the stamp after printing can be easily removed by a piece of office tape. Alternatively, the cubes can also be retrieved and reused by ultrasonicating the stamp for $5 \mathrm{~min}$ in the very same solvent in which the cubes were dispersed. After these steps, the stamp is very clean and can be reused. It is critical to make highly uniform stamps in terms of thickness. Uniform thickness ensures even distribution of pressure on the entire area and in turn high quality of the printed patterns.

Figure 2 shows a variety of nanopatterns formed using the imprinting process with a $\mathrm{Ag}$ nanocube resist. We can direct the cubes from the disordered state in the resist (Figure S6) to assembled curved profiles with nearly full circles and almost perfect face-to-face stacking along the circumference, as shown in Figure 2a,b. It is also possible to print curves with different radii of curvature $(\varphi)$. We were able to print curves with $\varphi=4$ $\mu \mathrm{m}$, all the way down to $300 \mathrm{~nm}$, as shown in Figure 2a,b and Figures S10-14. Additionally, it is possible to control the line width of the printed patterns with digital accuracy, for example, from 1 to 4 cubes as shown in Figure 2c,d,e,f. The number of cubes forming the width of the patterns can be simply controlled by tuning the trench width in the PDMS stamp. We also observed that even for the same trench width different printed line widths could be obtained by adjusting the applied pressure, although this was not explored systematically. Apart from curved profiles, it is also possible to print straight lines with this technique, for example, grids and gratings as shown in Figure 2g,h and Figures S15 and S16. In order to print assemblies of arbitrary $2 \mathrm{D}$ patterns, along with curves and lines, it is equally important to be able to print complex nodes and junctions. For demonstration, we have printed two-way, four-way, and three-way junctions as well as a sharp $90^{\circ}$ turn, as shown in Figure 2i,j,k,l, respectively (more examples in Figure S17). Finally, arrays of isolated nanocubes were printed with good orientational alignment (Figure $2 \mathrm{~m}, \mathrm{n}$ ), which can be interesting for applications using surface lattice resonances. ${ }^{59,60}$

The residual number of cubes in and around the desired patterns shown in Figure 2 and the corresponding supporting figures is really low. This is a significant result given that we have a few hundred million cubes $\left(2.1 \times 10^{11}\right.$ cubes $/ \mathrm{mL}$ for $\mathrm{Ag}$ nanocubes) to begin with at the resist stage before patterning. Such clean printing $(98.7 \%$ of nanocubes in the desired pattern) is possible because of two main reasons: First, a thin layer of solvent is present during printing, which provides sufficient in-plane motion for the cubes. Second, the flexible nature of the PDMS permits conformal and complete flat contact with the underlying substrate, pushing the excess cubes into the trenches. ${ }^{24}$

Nanocube imprint lithography can be easily implemented on a large scale. We have successfully printed patterns such as arrays of circles, gratings, and grids in areas on the order of $\mathrm{mm}^{2}$ on a Si substrate, as shown in Figure 3 and Figure S18. The printing area in this work is limited to $\mathrm{mm}^{2}$ only because 
of the Si master that was used to make the PDMS template. In principle, the process should be able to yield patterns at the wafer scale or beyond in a single print. The inset of Figure $3 c$ shows the $\mathrm{Si}$ substrate from which the optical image was acquired. The uniform bright colors arising from diffraction over the $2 \mathrm{~mm}^{2}$ square confirms the presence of the printed grid over the entire printed area.

The Ag nanocube ink contains excess PVP to preserve the colloidal stability of the particles. During the drying step, as the solvent evaporates, this excess PVP concentrates and packs around the cubes. After drying, it is retained on the substrate along with the cubes, forming a fairly even film (resist) owing to surface activation of the substrate. The Ag nanocube resist is essentially a composite of $\mathrm{Ag}$ cubes embedded in the matrix of PVP. The PVP cushion prevents the irreversible binding of the cubes to the substrate irrespective of the high surface energy of the substrate. This is the key to nanocube imprint lithography. The thin layer of solvent (ethanol) available due to rewetting can partially redisperse the PVP to allow for enhanced surface mobility. For rewetting, it is critical to choose a solvent that is good for PVP. For example, we rewetted the resist layer with dimethylformamide (DMF), which is another excellent solvent for PVP, and it also led to good patterning results (Figure S19a,b). In contrast, rewetting with a bad solvent for PVP such as toluene or hexane did not lead to any patterning (Figure S19c,d and e,f, respectively). Thus, during stamping, the viscous drag provided by the PVP/good solvent duo can move the cubes over substantial distances. Our results suggest that the Ag cubes can travel at least $10 \mu \mathrm{m}$ (see diagonally located circles of Figure S18d,e) and even up to $25 \mu \mathrm{m}$ (Figure S17e) in some cases.

Fundamentally, the patterning and packing are both governed by the confinement that is imposed by the trenches carved in PDMS. Patterning is the direct result of nanoparticles being driven to the trenches while pressing the stamp on the substrate, and the density of particles along with interparticle attraction determines the packing. The temporary binding and release of particles from the substrate as well as the displacement of particles on the substrate is facilitated by the $\mathrm{PVP} /$ ethanol combination (component forming the matrix and solvent compatible with it). Thus, if the native ink solution does not contain PVP, patterning can still be achieved by adsorbing a layer of PVP on the substrate before dispensing the desired nanoparticle ink. As a result, the process is not restricted to metallic Ag or cubic shape only. It can also nicely print other classes of materials as well as nanoparticles with different shapes, sizes, and surface ligand capping and dispersed in various solvents. For example, the gratings shown in the optical image of Figure $3 \mathrm{~b}$ and Figure S20 were printed with commercially available $50 \mathrm{~nm}$ silica nanospheres. The areas outside the lines are exceptionally clean with no residual particles. The spheres were originally dispersed in ethanol with silanol as the surface functional group. Before dropping the ink, the substrate was adsorbed with $2 \mu \mathrm{L}$ of $1 \mathrm{mM}$ PVP in ethanol and allowed to dry completely. We also tried $0.5,1,2$, and $4 \mu \mathrm{L}$ of PVP solution with constant area of the substrate, and beyond $1 \mu \mathrm{L}$ there was no perceivable difference in the printing results. The technique was also tested with other materials including $100 \mathrm{~nm}$ polystyrene spheres with carboxylate capping, $50 \mathrm{~nm} \mathrm{Au}$ nanocubes with citrate capping, and $20 \mathrm{~nm}$ Pd nanocubes with PVP capping. The results are shown in Figure S21, and they prove that the concept of nanocube imprint lithography can be translated to colloidal inks with different materials, shapes, sizes, and surface chemistry. Since the technique requires very little ink (a few $\mu \mathrm{L}$ per print), it makes the process efficient and economical from a technological standpoint, while the broad applicability is appealing for the field of nanopatterning.

Another interesting aspect of imprint lithography explored in this work was the ability to print high aspect ratio shapes. We were able to print curves and lines of a maximum aspect ratio of 5 as shown in Figure $4 a, b$ and Figure S22. The cubes are

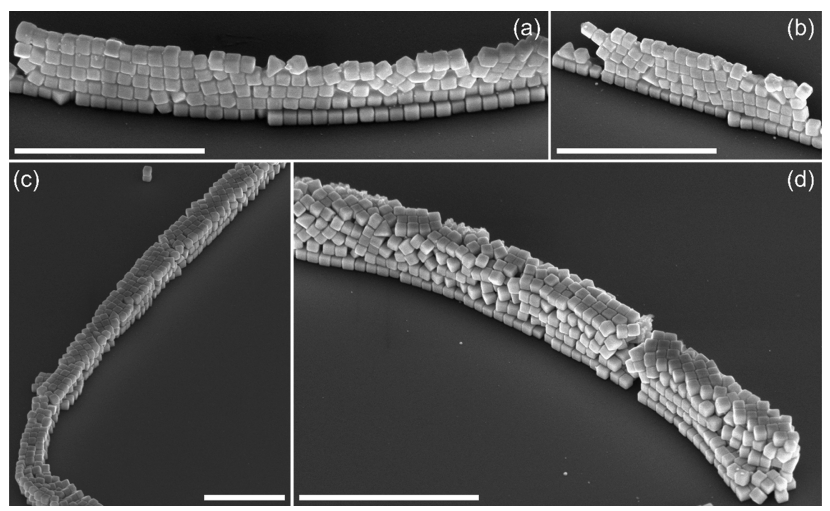

Figure 4. Patterns of (a) a curve and (b) a line with a maximum aspect ratio of 5 in the $z$-direction. (c, d) 3D patterns assembled out of $\mathrm{Ag}$ cubes. All scale bars are $1 \mu \mathrm{m}$ in length.

nicely sitting on top of each other and glued together with the interparticle force of attraction. The flat faces of the cubes lend the desired stability and allow for printing of single-particlewide yet 5 cubes tall free-standing walls. Such high aspect ratios are nearly impossible to attain with spherical or irregular particles, which is the most common shape found in colloidal inks. A previous report showed excellent layer-by-layer assembled 3 particle tall superlattices; however, it was achieved with top-down lithography on a specially engineered substrate using nanocrystals and DNA. ${ }^{61}$ As with the $2 \mathrm{D}$ patterns, the packing among the cubes in $3 \mathrm{D}$ is reasonably good, except where an irregularly shaped particle disturbs the flow. However, the process appears to be tolerant of particles of different shapes and sizes; for example, a tetrahedron appears in the second row from the bottom in both the patterns in Figure $4 a, b$, but the packing as a whole is robust (also see Figure $2 \mathrm{a}, \mathrm{e}, \mathrm{f})$. The digital line width control seen with $2 \mathrm{D}$ patterns also can be extended to $3 \mathrm{D}$ patterns, as shown in Figure $4 c$,d. Finally, the pattern quality can almost certainly be improved with further optimization of the imprinting press and process parameters.

\section{CONCLUSIONS}

We present a facile and reproducible technique that can print structures with nanoscale spatial resolution over large areas in a single step using nanocubes directly as an imprint resist. The technique was successfully applied to colloidally stable $\mathrm{Ag}$ nanocubes to print high-resolution curves, lines, grids, and complex junctions. We have also demonstrated single-particle lattices as well as 3D patterning, with tall patterns showing an aspect ratio up to 5, along with digital control over the line width. The topographically patterned PDMS stamps can be reused multiple times, and the elastic nature of the material facilitates clean printing of the patterns. Imprint lithography is a physical process, and we have demonstrated that it can be 
applied to a variety of materials and particles of different shapes and sizes, thus broadening the scope of the process. Additionally, it is inexpensive and fast and has the potential of on-chip patterning and device fabrication. We believe that flat printing can be easily translated to rolling with engineering optimizations. This work is a stepping stone for roll-to-roll integration that could prove very useful for industry and has ample potential for commercialization.

\section{METHODS}

Chemicals. $1 H, 1 H, 2 H, 2 H$-Perfluorooctyltriethoxysilane (98\%) (MW $510.36 \mathrm{~g} / \mathrm{mol}$ ); polyvinylpyrrolidone (average MW $55000 \mathrm{~g}$ ) $\mathrm{mol}$ and $29000 \mathrm{~g} / \mathrm{mol})$; silver nitrate $\left(\mathrm{AgNO}_{3}\right)(\mathrm{MW} 169.8 \mathrm{~g} / \mathrm{mol})$; copper chloride $\left(\mathrm{CuCl}_{2}\right)(\mathrm{MW} 170.48 \mathrm{~g} / \mathrm{mol}) ; 1,5$-pentanediol (MW $104.15 \mathrm{~g} / \mathrm{mol}$ ); ascorbic acid (AA) (MW $176.12 \mathrm{~g} / \mathrm{mol}$ ); potassium bromide (KBr) (MW $119 \mathrm{~g} / \mathrm{mol}$ ); sodium tetrachloropalladate $\left(\mathrm{Na}_{2} \mathrm{PdCl}_{4}\right.$ ) (MW $294.21 \mathrm{~g} / \mathrm{mol}$ ); Milli-Q water (resistivity -18 $\mathrm{M} \Omega \cdot \mathrm{cm}$ at $25{ }^{\circ} \mathrm{C}$ ) taken from a commercial purifier from Merck named Simplicity Water Purification System; acetone (ACS reagent, $>99.5 \%$ (GC), MW $58.08 \mathrm{~g} / \mathrm{mol}$ (VWR)); 2-propanol (IPA) (meets analytical specification, $>99.5 \%$ (GC), MW $60.10 \mathrm{~g} / \mathrm{mol}$ (VWR)); ethanol (>99.5\% Ph. Eur., MW $46.07 \mathrm{~g} / \mathrm{mol}(\mathrm{VWR})$ ); tetramethylammonium hydroxide solution (TMAH) (25 wt \% in water, MW $91.15 \mathrm{~g} / \mathrm{mol}$ ); silica nanospheres $50 \mathrm{~nm}$ (lot: MKCJ8723); latex beads polystyrene (lot: MKCJ1534); and gold nanocubes with citrate capping (50 nm, lot: J6911) from nanopartz.com were used. All chemicals were purchased from Sigma-Aldrich unless stated otherwise and used as received without any further processing. Elastosil RT 601 A/B (RTV-2 silicone rubber) PDMS was bought from Wacker Chemie.

Silicon Substrates. Si substrates of $12 \times 12 \mathrm{~mm}$ were cut from a 4 in. boron-doped $\langle 100\rangle$ orientation single-crystal wafer purchased from Siegert Wafer GmbH, Aachen, Germany, and used for Si master fabrication.

Synthesis of Ag Nanocubes. A $5 \mathrm{~mL}$ solution of $0.235 \mathrm{M}$ $\mathrm{AgNO}_{3}$ in pentanediol was mixed with $40 \mu \mathrm{L}$ of $0.043 \mathrm{M} \mathrm{CuCl}_{2}$ and ultrasonicated for about $30 \mathrm{~min}$ until the solution turned turbid yellow-orange. PVP (MW $55000 \mathrm{~g} / \mathrm{mol}$ ) with a concentration of 40 $\mathrm{g} / \mathrm{L}$ was prepared in pentanediol in a separate vial (ultrasonicated for fast dissolution). A round-bottom flask was suspended in a silicone oil bath at $195^{\circ} \mathrm{C}$ with $10 \mathrm{~mL}$ of pentanediol. After 10 min of preheating, $500 \mu \mathrm{L}$ of a $\mathrm{AgNO}_{3}$ and $\mathrm{CuCl}_{2}$ precursor mixture was added in one go immediately followed by $490 \mu \mathrm{L}$ of dropwise injection of PVP. After $30 \mathrm{~s}$ of precursor injection, another $490 \mu \mathrm{L}$ of PVP was injected dropwise in the reaction flask. This marks the end of the first cycle. Exactly $60 \mathrm{~s}$ after the first precursor injection begins the second cycle of injections and 5 complete cycles in total to make very well-defined (100) faceted cubes of $75 \mathrm{~nm}$. The reaction mixture goes through a sequence of color changes, and eventually, it turns creamy light green. The solution was stirred at $300 \mathrm{rpm}$, and the flask was open to the atmosphere for the entire time. Finally, after the fifth cycle, it was quenched in a normal water bath. A series of centrifugations along with coarse and fine filtration was performed to isolate cubes from the rest of the byproducts of the synthesis. The $\mathrm{Ag}$ cubes were synthesized by adopting a modified procedure reported previously. $^{42,62}$

A major part of the experiments in this work was done with $\mathrm{Ag}$ cubes bought from nanoComposix, Inc. (nanocomposix.com).

Synthesis of Pd Nanocubes. The cubes were synthesized according to a previous report. ${ }^{63} \mathrm{~A} 105 \mathrm{mg}$ amount of PVP (55k), 60 $\mathrm{mg}$ of $\mathrm{AA}, 600 \mathrm{mg}$ of $\mathrm{KBr}$, and $8 \mathrm{~mL}$ of Milli-Q water were taken in a $20 \mathrm{~mL}$ vial. A stir bar was also added. The vial was placed in a preheated oil bath at $80{ }^{\circ} \mathrm{C}$ and stirred at $300 \mathrm{rpm}$. The solution turned colorless in a few minutes. Meanwhile, $57 \mathrm{mg}$ of $\mathrm{Na}_{2} \mathrm{PdCl}_{4}$ in 3 $\mathrm{mL}$ of Milli-Q water was dissolved in a separate vial, and after $10 \mathrm{~min}$, this solution was injected in the main reaction vial rapidly. The vial was capped and also wrapped with Parafilm, and the reaction was allowed to run for $3 \mathrm{~h}$. The solution eventually turned dark brown. The vial was allowed to cool by air convection, and later the product was collected by centrifugation. The Pd cubes were finally dispersed in ethanol for subsequent use.

Si Master Fabrication. Exposure. EBL was used to make nanopatterns on the Si substrate. The instrument used was an eLINE from Raith Nanofabrication GmbH installed in an ISO 7 Class 10000 cleanroom at AMOLF. The complete fabrication of the Si master was carried out in the cleanroom at AMOLF.

A $12 \times 12 \mathrm{~mm}$ Si piece cut from a $3 \mathrm{in}$. Si single crystal wafer was cleaned with acetone, IPA, and plasma $\left(\mathrm{O}_{2}\right.$ descum recipe $)$ for $10 \mathrm{~s}$ with $50 \mathrm{~W}$ forward power oxygen plasma in a Plasmalab $80+$ from Oxford Instruments and then immediately spin coated (without a spinning bowl on top) with hydrogen silsesquioxane (HSQ) resist in a Delta 80 spin coater from Suss MicroTec. The resist was dropped from a syringe until it completely covered the $S i$ substrate and then spun at $3200 \mathrm{rpm}$, with $800 \mathrm{rpm} / \mathrm{s}$ acceleration for $45 \mathrm{~s}$, which resulted in a $110 \mathrm{~nm}$ thick HSQ-coated $\mathrm{Si}$ substrate. Then it was baked at $180^{\circ} \mathrm{C}$ for $120 \mathrm{~s}$. Subsequently, this substrate was exposed to an e-beam with a dose of $4000 \mu \mathrm{C} / \mathrm{cm}^{2}$ with circles of varying radii $(300 \mathrm{~nm}, 500 \mathrm{~nm}, 1 \mu \mathrm{m}, 2 \mu \mathrm{m}$, and $4 \mu \mathrm{m}$ ) and widths (100 and 200 $\mathrm{nm})$. The column mode used for exposure was LC $60 \mu \mathrm{m}$ with the area and curved elements exposure mode and step size of $5 \mathrm{~nm}$ along with beam current of $0.5 \mathrm{nA}$. A similar procedure was followed to make the $\mathrm{Si}$ master with patterned ridges in the form of grids or gratings for imprinting.

Development. After exposure, it was developed in TMAH at $50{ }^{\circ} \mathrm{C}$ for $60 \mathrm{~s}$ and then rinsed twice thoroughly with water. This step dissolves all the unexposed resist, leaving behind the nanopatterns of HSQ in the form of ridges on the Si substrate ( $\mathrm{Si}$ master) as shown in Figure S2.

Etching. In order to make deeper trenches in the PDMS, the Si master was etched to the desired height (Figure S3). The patterned lines of HSQ are made taller by plasma etching the Si substrate using the remaining resist in the form of patterns as the mask in COBRA (Oxford Instruments). The Si substrate is attached to a carrier wafer ( 4 in. $\mathrm{Si}$ ) by Fomblin oil. The oil is used to improve the thermal conductivity between the interface of the substrate and the wafer. For etching, a $\mathrm{Cl}_{2}+\mathrm{HBr} / \mathrm{O}_{2}$ recipe is used where the initial $11 \mathrm{~s}$ of $\mathrm{Cl}_{2}$ is used to remove the oxide layer on the $\mathrm{Si}$ and then the virgin $\mathrm{Si}$ is etched deeper by $\mathrm{HBr} / \mathrm{O}_{2}$ for another $4 \mathrm{~min}$. The etching is carried out at $60{ }^{\circ} \mathrm{C}$ with a $\mathrm{Cl}_{2}$ flow rate of $50 \mathrm{sccm}$, which yielded ridges of $550 \mathrm{~nm}$ in height.

Masters that did not require etching were directly moved to the silanization step. The etched ones, before silanization, were subjected to a HF bath ( $2 \%$ concentration) for $15 \mathrm{~min}$ to remove the residual HSQ from the top of the ridges on the Si substrate.

Silanization. After development, if not subjected to etching, the $\mathrm{Si}$ master is baked at $200{ }^{\circ} \mathrm{C}$ on a hot plate for $30 \mathrm{~min}$. Following this, it is again plasma treated for $10 \mathrm{~s}$ and placed beside $20 \mu \mathrm{L}$ of $1 \mathrm{H}, 1 \mathrm{H}, 2 \mathrm{H}, 2 \mathrm{H}$-perfluorooctyltriethoxysilane in a Teflon boat at $50{ }^{\circ} \mathrm{C}$ in a Vacucenter VC20 vacuum oven from Salvis LAB, Switzerland, at $50 \mathrm{mbar}$ for $30 \mathrm{~min}$. This treatment forms a self-assembled monolayer of fluorinated silane chains on the surface of the Si master and renders it highly hydrophobic. This facilitates peeling off the cured PDMS from the Si master.

PDMS Stamp Preparation. The PDMS stamp is prepared by mixing monomer and polymerizing agent in a ratio of 9:1. After thorough mixing and degassing (to eliminate air bubbles), the liquid mixture is poured onto a patterned F-silanized $\mathrm{Si}$ master. It is backed by a UV-ozone-activated ( $45 \mathrm{~min}$ in a UV ozone ProCleaner from BioForce Nanosciences) glass slide and then cured in an oven at 80 ${ }^{\circ} \mathrm{C}$ for $24 \mathrm{~h}$. A Viton gasket made by a laser cutter (Speedy 400 from Trotec Laser B.V.) was used as the spacer layer to define the dimensions of the PDMS. This exercise results in a PDMS stamp as shown in Figure S4. The PDMS slab is $4 \times 4 \times 1 \mathrm{~mm}$ in dimensions and has nanotrenches on one side and bonded covalently to the glass slide on the other side.

Printing Experiments. Preparation of the Si Substrate for Imprint Lithography. A Si wafer was spin coated with Microposit S1813 positive resist at $4000 \mathrm{rpm}$ at the rate of $1000 \mathrm{rpm} / \mathrm{s}$ acceleration for $45 \mathrm{~s}$. This resulted in a $1.3 \mu \mathrm{m}$ thick resist layer that 
was baked at $115^{\circ} \mathrm{C}$ for $60 \mathrm{~s}$. This wafer was then exposed to a dose of $140 \mathrm{~mJ} / \mathrm{cm}^{2}\left(25 \mathrm{~mJ} / \mathrm{cm}^{2}\right.$ for $\left.5.6 \mathrm{~s}\right)$ in the hard contact mode in an MABA6 mask aligner from SUSS MicroTec SE (Germany) using the foil mask shown in Figure S5a. The mask was printed with a laser printer on a transparency and then manually overwritten with a permanent marker on the printed $5 \mathrm{~mm}$ spots. Then the transparency was stuck to a quartz plate with sticky tape and used as is for exposure. After exposure, the wafer was developed in Microposit MF-319 for 60 $\mathrm{s}$ and then rinsed with water. This results in a wafer as shown in Figure S5b.

This Si wafer was further silanized and made hydrophobic as aforementioned in the Si Master Fabrication section. This made the Si surface hydrophobic everywhere except the center $5 \mathrm{~mm}$ dot covered with resist. Subsequently, the wafer was diced along the straight lines to make $10 \times 10 \mathrm{~mm} \mathrm{Si}$ substrates used for imprinting.

Imprinting. The printing experiments were carried out with the help of a Universal Testing System model 5965 with $50 \mathrm{kN}$ force capacity from Instron, MA, USA. A custom holder was made to mount the PDMS stamp and the Si substrate with the clamps of the machine, as shown in Figure S7a,b. The holder was also equipped with a Peltier element to heat or cool the sample stage as required. The stamp and the $\mathrm{Si}$ substrate were held in place on the respective holders via vacuum.

The $10 \times 10 \mathrm{~mm} \mathrm{Si} \mathrm{substrate} \mathrm{was} \mathrm{ultrasonicated} \mathrm{in} \mathrm{acetone} \mathrm{and}$ IPA for $60 \mathrm{~s}$ each to clean the resist. Subsequently, it was activated by UV-ozone treatment for $30 \mathrm{~min}$ and then mounted on the holder. A 1 $\mu \mathrm{L}$ amount of the colloidal $\mathrm{Ag}$ cubes (or the desired ink) dispersed in ethanol was dispensed on the activated substrate and allowed to dry to form a film of cubes on the substrate. After complete drying, $3 \mu \mathrm{L}$ of pure ethanol was added to the same spot as the cubes (rewetting on the PDMS stamp instead of the sample works equally well), and the stamp was pressed on the substrate at the rate of $1 \mathrm{~mm} / \mathrm{min}$ until a force of $10-30 \mathrm{~N}$. The solvent was allowed to dry (either overnight or by heating the substrate to $40{ }^{\circ} \mathrm{C}$ ) before the stamp was retracted at the rate of $10 \mu \mathrm{m} / \mathrm{min}$ to $1 \mathrm{~mm} / \mathrm{min}$.

Excess cubes stuck to the surface of the stamp or in the trenches after printing can be easily removed (or retrieved) by ultrasonicating the stamp for $5 \mathrm{~min}$ in the same solvent in which the cubes were dispersed. After this step, the stamp is practically very clean and can be reused again and again by applying the same cleaning protocol. The concentration of the inks was adjusted to $1 \mathrm{mg} / \mathrm{mL}$.

Characterization. Light Optical Microscopy. Optical images were taken with an Axio Imager.A2m (Carl Zeiss B.V., Netherlands) and Eclipse LV100 ND (Nikon Instruments, Netherlands).

Scanning Electron Microscopy (SEM). High-resolution SEM images were acquired using an FEI Verios 460 with an acceleration voltage and current of $5 \mathrm{kV}$ and $100 \mathrm{pA}$, respectively.

\section{ASSOCIATED CONTENT}

\section{s) Supporting Information}

The Supporting Information is available free of charge at https://pubs.acs.org/doi/10.1021/acsnano.0c04793.

List of a few key points to ensure good fidelity of the process, images of Ag cubes and resist, Si master, PDMS stamp; details about various stamps used and the imprinting press; additional SEM images (PDF)

Video of the process (MP4)

\section{AUTHOR INFORMATION}

\section{Corresponding Author}

Erik C. Garnett - Center for Nanophotonics, AMOLF, 1098 XG Amsterdam, The Netherlands; 이이. orcid.org/0000-00029158-8326; Email: garnett@amolf.nl

\section{Author}

Harshal Agrawal - Center for Nanophotonics, AMOLF, 1098 XG Amsterdam, The Netherlands; 10 orcid.org/0000-00025106-3947

Complete contact information is available at: https://pubs.acs.org/10.1021/acsnano.0c04793

\section{Notes}

The authors declare no competing financial interest.

\section{ACKNOWLEDGMENTS}

The work at AMOLF is part of the research program of the "Nederlandse Organisatie voor Wetenschappelijk Onderzoek" (NWO). This work was supported by the NWO VIDI grant (project number 14846). The authors would like to sincerely thank Bas Overvelde for letting us use his laboratory and the equipment for experiments and Niels Commandeur, Dion Ursem, and Marc Duursma for all the technical support. The help from Agustin Iniguez Rabago to acquire a camera image of the Si substrate with printed grids in Figure ${ }^{3}$ (inset); HenkJan Boluijt and Lukas Engel for the schematic in Figure ${ }^{1}$; and Jian-Yao Zheng for the video is gratefully acknowledged. The authors thank Esther Alarcon-Llado for carefully reading and commenting on the manuscript.

\section{REFERENCES}

(1) Pimpin, A.; Srituravanich, W. Review on Micro- and Nanolithography Techniques and Their Applications. Eng. J. 2012, 16, 3756.

(2) Choi, J.-H.; Wang, H.; Oh, S. J.; Paik, T.; Sung, P.; Sung, J.; Ye, X.; Zhao, T.; Diroll, B. T.; Murray, C. B.; Kagan, C. R. Exploiting the Colloidal Nanocrystal Library to Construct Electronic Devices. Science 2016, 352, 205-208.

(3) Kagan, C. R.; Lifshitz, E.; Sargent, E. H.; Talapin, D. V. Building Devices from Colloidal Quantum Dots. Science 2016, 353, No. aac5523-1.

(4) Kumar, A.; Whitesides, G. M. Features of Gold Having Micrometer to Centimeter Dimensions Can Be Formed through a Combination of Stamping with an Elastomeric Stamp and an Alkanethiol "Ink"' Followed by Chemical Etching. Appl. Phys. Lett. 1993, 63, 2002-2004.

(5) Wilbur, J. L.; Kumar, A.; Kim, E.; Whitesides, G. M. Microfabrication by Microcontact Printing of Self-Assembled Monolayers. Adv. Mater. 1994, 6, 600-604.

(6) Kumar, A.; Biebuyck, H. A.; Whitesides, G. M. Patterning SelfAssembled Monolayers: Applications in Materials Science. Langmuir 1994, 10, 1498-1511.

(7) Liu, X.; Fu, L.; Hong, S.; Dravid, V. P.; Mirkin, C. A. Arrays of Magnetic Nanoparticles Patterned via "Dip-Pen" Nanolithography. Adv. Mater. 2002, 14, 231-234.

(8) Yang, P.; Zhang, L.; Kang, D. J.; Strahl, R.; Kraus, T. HighResolution Inkjet Printing of Quantum Dot Light-Emitting Microdiode Arrays. Adv. Opt. Mater. 2020, 8, 1901429.

(9) Liu, S.; Maoz, R.; Sagiv, J. Planned Nanostructures of Colloidal Gold via Self-Assembly on Hierarchically Assembled Organic Bilayer Template Patterns with In-Situ Generated Terminal Amino Functionality. Nano Lett. 2004, 4, 845-851.

(10) Garcia, R.; Knoll, A. W.; Riedo, E. Advanced Scanning Probe Lithography. Nat. Nanotechnol. 2014, 9, 577-587.

(11) Flauraud, V.; Mastrangeli, M.; Bernasconi, G. D.; Butet, J.; Alexander, D. T. L.; Shahrabi, E.; Martin, O. J. F.; Brugger, J. Nanoscale Topographical Control of Capillary Assembly of Nanoparticles. Nat. Nanotechnol. 2017, 12, 73-80.

(12) Ni, S.; Isa, L.; Wolf, H. Capillary Assembly as a Tool for the Heterogeneous Integration of Micro- and Nanoscale Objects. Soft Matter 2018, 14, 2978-2995. 
(13) Kraus, T.; Malaquin, L.; Schmid, H.; Riess, W.; Spencer, N. D.; Wolf, H. Nanoparticle Printing with Single-Particle Resolution. Nat. Nanotechnol. 2007, 2, 570-576.

(14) Chou, S. Y.; Krauss, P. R.; Renstrom, P. J. Imprint Lithography with 25-Nanometer Resolution. Science 1996, 272, 85-87.

(15) Chou, S. Y.; Krauss, P. R. Imprint Lithography with Sub-10 nm Feature Size and High Throughput. Microelectron. Eng. 1997, 35, 237-240.

(16) Austin, M. D.; Ge, H.; Wu, W.; Li, M.; Yu, Z.; Wasserman, D.; Lyon, S. A.; Chou, S. Y. Fabrication of $5 \mathrm{~nm}$ Linewidth and $14 \mathrm{~nm}$ Pitch Features by Nanoimprint Lithography. Appl. Phys. Lett. 2004, 84, 5299-5301.

(17) Chou, S. Y.; Keimel, C.; Gu, J. Ultrafast and Direct Imprint of Nanostructures in Silicon. Nature 2002, 417, 835-837.

(18) Henzie, J.; Barton, J. E.; Stender, C. L.; Odom, T. W. LargeArea Nanoscale Patterning: Chemistry Meets Fabrication. Acc. Chem. Res. 2006, 39, 249-257.

(19) Verschuuren, M. A.; Megens, M.; Ni, Y.; van Sprang, H.; Polman, A. Large Area Nanoimprint by Substrate Conformal Imprint Lithography (SCIL). Adv. Opt. Technol. 2017, 6, 243-264.

(20) Tan, H. Roller Nanoimprint Lithography. J. Vac. Sci. Technol, B: Microelectron. Process. Phenom. 1998, 16, 3926.

(21) Ahn, S. H.; Guo, L. J. High-Speed Roll-To-Roll Nanoimprint Lithography on Flexible Plastic Substrates. Adv. Mater. 2008, 20, 2044-2049.

(22) Ahn, S. H.; Guo, L. J. Large-Area Roll-To-Roll and Roll-ToPlate Nanoimprint Lithography: A Step toward High-Throughput Application of Continuous Nanoimprinting. ACS Nano 2009, 3, 2304-2310.

(23) Kothari, R.; Beaulieu, M. R.; Hendricks, N. R.; Li, S.; Watkins, J. J. Direct Patterning of Robust One-Dimensional, Two-Dimensional, and Three-Dimensional Crystalline Metal Oxide Nanostructures Using Imprint Lithography and Nanoparticle Dispersion Inks. Chem. Mater. 2017, 29, 3908-3918.

(24) King, E.; Xia, Y.; Zhao, X.-M.; Whitesides, G. M. SolventAssisted Microcontact Molding: A Convenient Method for Fabricating Three-Dimensional Structures on Surfaces of Polymers. Adv. Mater. 1997, 9, 651-654.

(25) Beaulieu, M. R.; Hendricks, N. R.; Watkins, J. J. Large-Area Printing of Optical Gratings and 3D Photonic Crystals Using Solution-Processable Nanoparticle/Polymer Composites. ACS Photonics 2014, 1, 799-805.

(26) Li, W.; Zhou, Y.; Howell, I. R.; Gai, Y.; Naik, A. R.; Li, S.; Carter, K. R.; Watkins, J. J. Direct Imprinting of Scalable, HighPerformance Woodpile Electrodes for Three-Dimensional LithiumIon Nanobatteries. ACS Appl. Mater. Interfaces 2018, 10, 5447-5454.

(27) Lee, M. H.; Huntington, M. D.; Zhou, W.; Yang, J.-C.; Odom, T. W. Programmable Soft Lithography: Solvent-Assisted Nanoscale Embossing. Nano Lett. 2011, 11, 311-315.

(28) Odom, T. W.; Love, J. C.; Wolfe, D. B.; Paul, K. E.; Whitesides, G. M. Improved Pattern Transfer in Soft Lithography Using Composite Stamps. Langmuir 2002, 18, 5314-5320.

(29) Xia, Y.; Whitesides, G. M. Soft Lithography. Annu. Rev. Mater. Sci. 1998, 28, 153-184.

(30) Ding, I.-K.; Zhu, J.; Cai, W.; Moon, S.-J.; Cai, N.; Wang, P.; Zakeeruddin, S. M.; Grätzel, M.; Brongersma, M. L.; Cui, Y.; McGehee, M. D. Plasmonic Dye-Sensitized Solar Cells. Adv. Energy Mater. 2011, 1, 52-57.

(31) Spinelli, P.; Verschuuren, M. A.; Polman, A. Broadband Omnidirectional Antireflection Coating Based on Subwavelength Surface Mie Resonators. Nat. Commun. 2012, 3, 692.

(32) He, X.; Liu, P.; Zhang, H.; Liao, Q.; Yao, J.; Fu, H. Patterning Multicolored Microdisk Laser Arrays of Cesium Lead Halide Perovskite. Adv. Mater. 2017, 29, 1604510.

(33) Zhang, H.; Liao, Q.; Wu, Y.; Zhang, Z.; Gao, Q.; Liu, P.; Li, M.; Yao, J.; Fu, H. 2D Ruddlesden-Popper Perovskites Microring Laser Array. Adv. Mater. 2018, 30, 1706186.

(34) Matricardi, C.; Hanske, C.; Garcia-Pomar, J. L.; Langer, J.; Mihi, A.; Liz-Marzán, L. M. Gold Nanoparticle Plasmonic Super- lattices as Surface-Enhanced Raman Spectroscopy Substrates. ACS Nano 2018, 12, 8531-8539.

(35) van de Groep, J.; Gupta, D.; Verschuuren, M. A.; Wienk, M.; Janssen, R. A. J.; Polman, A. Large-Area Soft-Imprinted Nanowire Networks as Light Trapping Transparent Conductors. Sci. Rep. 2015, 5,11414 .

(36) Sciacca, B.; van de Groep, J.; Polman, A.; Garnett, E. C. Solution-Grown Silver Nanowire Ordered Arrays as Transparent Electrodes. Adv. Mater. 2016, 28, 905-909.

(37) Maurer, J. H. M.; González-García, L.; Reiser, B.; Kanelidis, I.; Kraus, T. Templated Self-Assembly of Ultrathin Gold Nanowires by Nanoimprinting for Transparent Flexible Electronics. Nano Lett. 2016, 16, 2921-2925.

(38) Maurer, J. H. M.; González-García, L.; Backes, I. K.; Reiser, B.; Schlossberg, S. M.; Kraus, T. Direct Nanoimprinting of a Colloidal Self-Organizing Nanowire Ink for Flexible, Transparent Electrodes. Adv. Mater. Technol. 2017, 2, 1700034.

(39) Ko, S. H.; Park, I.; Pan, H.; Grigoropoulos, C. P.; Pisano, A. P.; Luscombe, C. K.; Fréchet, J. M. J. Direct Nanoimprinting of Metal Nanoparticles for Nanoscale Electronics Fabrication. Nano Lett. 2007, 7, 1869-1877.

(40) Alam, M.; Cameron, D. Investigation of Annealing Effects on Sol-Gel Deposited Indium Tin Oxide Thin Films in Different Atmospheres. Thin Solid Films 2002, 420-421, 76-82.

(41) Kamyshny, A.; Magdassi, S. Conductive Nanomaterials for Printed Electronics. Small 2014, 10, 3515-3535.

(42) Sciacca, B.; Berkhout, A.; Brenny, B. J. M.; Oener, S. Z.; van Huis, M. A.; Polman, A.; Garnett, E. C. Monocrystalline Nanopatterns Made by Nanocube Assembly and Epitaxy. Adv. Mater. 2017, 29, 1701064 .

(43) Agrawal, H.; Patra, B. K.; Altantzis, T.; De Backer, A.; Garnett, E. C. Quantifying Strain and Dislocation Density at Nanocube Interfaces after Assembly and Epitaxy. ACS Appl. Mater. Interfaces 2020, 12, 8788-8794.

(44) Yang, H. J.; He, S. Y.; Chen, H. L.; Tuan, H. Y. Monodisperse Copper Nanocubes: Synthesis, Self-Assembly, and Large-Area DensePacked Films. Chem. Mater. 2014, 26, 1785-1793.

(45) Lagrow, A. P.; Ingham, B.; Cheong, S.; Williams, G. V. M.; Dotzler, C.; Toney, M. F.; Jefferson, D. A.; Corbos, E. C.; Bishop, P. T.; Cookson, J.; Tilley, R. D. Synthesis, Alignment, and Magnetic Properties of Monodisperse Nickel Nanocubes. J. Am. Chem. Soc. 2012, 134, 855-858.

(46) Tao, A. R.; Habas, S.; Yang, P. Shape Control of Colloidal Metal Nanocrystals. Small 2008, 4, 310-325.

(47) Koczkur, K. M.; Mourdikoudis, S.; Polavarapu, L.; Skrabalak, S. E. Polyvinylpyrrolidone (PVP) in Nanoparticle Synthesis. Dalt. Trans. 2015, 44, 17883-17905.

(48) Park, J.-E.; Lee, Y.; Nam, J.-M. Precisely Shaped, Uniformly Formed Gold Nanocubes with Ultrahigh Reproducibility in SingleParticle Scattering and Surface-Enhanced Raman Scattering. Nano Lett. 2018, 18, 6475-6482.

(49) Macpherson, H. A.; Stoldt, C. R. Iron Pyrite Nanocubes: Size and Shape Considerations for Photovoltaic Application. ACS Nano 2012, 6, 8940-8949.

(50) Kuo, C.-H.; Chen, C.-H.; Huang, M. H. Seed-Mediated Synthesis of Monodispersed Cu2O Nanocubes with Five Different Size Ranges from 40 to $420 \mathrm{~nm}$. Adv. Funct. Mater. 2007, 17, 37733780.

(51) Protesescu, L.; Yakunin, S.; Bodnarchuk, M. I.; Krieg, F.; Caputo, R.; Hendon, C. H.; Yang, R. X.; Walsh, A.; Kovalenko, M. V. Nanocrystals of Cesium Lead Halide Perovskites (CsPbX3, X $=\mathrm{Cl}$, $\mathrm{Br}$, and I): Novel Optoelectronic Materials Showing Bright Emission with Wide Color Gamut. Nano Lett. 2015, 15, 3692-3696.

(52) Chen, H.-S.; Wu, S.-C.; Huang, M. H. Direct Synthesis of SizeTunable PbS Nanocubes and Octahedra and the PH Effect on Crystal Shape Control. Dalt. Trans. 2015, 44, 15088-15094.

(53) Wang, S.-B.; Min, Y.-L.; Yu, S.-H. Synthesis and Magnetic Properties of Uniform Hematite Nanocubes. J. Phys. Chem. C 2007, $111,3551-3554$. 
(54) Dang, F.; Mimura, K.; Kato, K.; Imai, H.; Wada, S.; Haneda, H.; Kuwabara, M. Growth of Monodispersed SrTiO3 Nanocubes by Thermohydrolysis Method. CrystEngComm 2011, 13, 3878-3883.

(55) Mai, H.-X.; Sun, L.-D.; Zhang, Y.-W.; Si, R.; Feng, W.; Zhang, H.-P.; Liu, H.-C.; Yan, C.-H. Shape-Selective Synthesis and Oxygen Storage Behavior of Ceria Nanopolyhedra, Nanorods, and Nanocubes. J. Phys. Chem. B 2005, 109, 24380-24385.

(56) Joshi, U. A.; Jang, J. S.; Borse, P. H.; Lee, J. S. Microwave Synthesis of Single-Crystalline Perovskite $\mathrm{BiFeO} 3$ Nanocubes for Photoelectrode and Photocatalytic Applications. Appl. Phys. Lett. 2008, 92, 242106.

(57) Yang, S.; Gao, L. Controlled Synthesis and Self-Assembly of CeO2 Nanocubes. J. Am. Chem. Soc. 2006, 128, 9330-9331.

(58) Lee, J. N.; Park, C.; Whitesides, G. M. Solvent Compatibility of Poly(dimethylsiloxane)-Based Microfluidic Devices. Anal. Chem. 2003, 75, 6544-6554.

(59) Juodenas, M.; Tamulevičius, T.; Henzie, J.; Erts, D.; Tamulevičius, S. Surface Lattice Resonances in Self-Assembled Arrays of Monodisperse Ag Cuboctahedra. ACS Nano 2019, 13, 9038-9047.

(60) Kravets, V. G.; Kabashin, A. V.; Barnes, W. L.; Grigorenko, A. N. Plasmonic Surface Lattice Resonances: A Review of Properties and Applications. Chem. Rev. 2018, 118, 5912-5951.

(61) Lin, Q.-Y.; Mason, J. A.; Li, Z.; Zhou, W.; O’Brien, M. N.; Brown, K. A.; Jones, M. R.; Butun, S.; Lee, B.; Dravid, V. P.; Aydin, K.; Mirkin, C. A. Building Superlattices from Individual Nanoparticles via Template-Confined DNA-Mediated Assembly. Science 2018, 359, 669-672.

(62) Sun, Y.; Xia, Y. Shape-Controlled Synthesis of Gold and Silver Nanoparticles. Science 2002, 298, 2176-2179.

(63) Chen, T.; Zhang, Y.; Xu, W. Size-Dependent Catalytic Kinetics and Dynamics of Pd Nanocubes: A Single-Particle Study. Phys. Chem. Chem. Phys. 2016, 18, 22494-22502. 